\title{
Effect of Plant Growth Regulators (GA3, NAA and BA) on Growth and Flowering of Gladiolus (Gladiolus hybridus Hort.) cv. White Prosperity
}

\author{
Anant Ram Singh ${ }^{1 *}$, Satendra Kumar ${ }^{1}, \operatorname{Rehan}^{2}$ and Deepti Jha ${ }^{3}$ \\ ${ }^{1}$ Department of Horticulture, College of Agriculture, Sardar Vallabhbhai Patel University of \\ Agri. and Tech. Meerut, Uttar Pradesh, India \\ ${ }^{2}$ Department of Horticulture, College of Agriculture, Jawaharlal Nehru Krishi \\ Vishwavidyalaya, Jabalpur, Madhya Pradesh, India \\ ${ }^{3}$ Department of Plant Pathology, College of Agriculture, Indira Gandhi Krishi \\ Vishwavidyalaya, Raipur, Chhattisgarh, India \\ *Corresponding author
}

\section{A B S T R A C T}

Keywords

Gladiolus, Plant growth regulators, Corm dipping, Spraying and corm dipping + foliar spraying

Article Info

Accepted:

17 January 2021

Available Online:

10 February 2021
The present investigation entitled "Effect of plant growth regulators on growth and flowering characters of gladiolus (Gladiolus hybridus Hort.) cv. White Prosperity" was conducted at Horticulture Research Center (HRC), College of Agriculture, Sardar Vallabhbhai Patel University of Agriculture \& Technology, Meerut-250110 (U.P.) during the rabi season of 2018-2019. The investigation revealed that plant growth regulators showed significant results on gladiolus as $\mathrm{GA}_{3}$ at $400 \mathrm{ppm}$ or $800 \mathrm{ppm}$ concentration was significantly superior to other treatments in improving the growth, days of sprouting, plant height, length of longest leaf, number of days required for visibility of first spike, days required for opening of first floret, diameter of spike, number of florets per spike, diameter of floret, longevity of spike. NAA at 200, 400 and 600 ppm increases the number of leaves per plant, plant height. When the different treatments of application were compared, it was found that corm dipping + foliar spraying treatment was significantly superior to control.

\section{Introduction}

Gladiolus (Gladiolus hybridus Hort.) is an ornamental cormelous plant native to South Africa. It belongs to monocot family Iridaceae and sub-family Ixioideae. Iridaceace family contains some 106 genera, containing mostly bulbous ornamentals. Gladiolus takes its name from latin word 'Gladius' because of sword like shape, therefore this is also known as 
'Sword lily'. Gladiolus is grown as flower bed in gardens and used in floral arrangements for interior decoration as well as making high quality bouquets (Lepcha et al., 2007).

Gladiolus is cultivated in most of the tropical and subtropical countries of the world. Its spikes takes 60 to 100 days after planting to be harvested depending upon the cultivars and time of year (Jenkins et al., 1970). The major gladiolus growing area in India are Kalimpong (West Bengal), New Delhi, Srinagar, Jammu \& Kashmir, Pune, Ludhiana, Bengaluru and Uttarakhand.

This phenomenal growth of floriculture in India during the last couple of decade has led the world floriculture experts to visualize for country as major player in floriculture trade in future.

To enhance yield and quality of any flower crop various cultural management practices like good planting material, suitable time of planting, spacing, irrigation included plant protection measure are required. The planting material corm is important factor, which governs the growth and development of gladiolus. Plant growth regulators or phytohormones are organic substances produced naturally in higher plants, controlling growth or other physiological functions at a site remote from its Place of production and active in minute amounts. The application of plant growth regulators is one of the most important factors in improving the growth, yield and flower quality (Nuvale et al., 2010).

Gibberellic acids has an important role in different plant processes, including seed germination, stem elongation, leaf expansion and flower development (Olszewski et al., 2002) and was found highly effective for increasing the sprouting percentage of corm, increased cormel production and cormel size in gladiolus (Padmalatha et al., 2013).

\section{Materials and Methods}

An experiment was conducted at Horticulture Research Centre of Sardar Vallabhbhai Patel University of Agriculture and Technology, Meerut (UP) during the rabi season (October 2018-April 2019). The experiment was laid out in randomized block design (RBD) with 28 treatments in three replications. The growth regulators viz., $\mathrm{GA}_{3}$, NAA and $\mathrm{BA}$ were used each at three different concentrations applied as corm dipping, foliar spraying and corm dipping + foliar spraying. A control of untreated corms was also maintained with three replications. The experimental field was prepared by repeated tractor plough followed by harrowing. Weeds and crop residue were removed and the land was levelled. Thus pulverized field was later divided into plots.

Irrigation channel, bunds and path were left around the experimental field according to the requirement. Analysis for estimation of growth and flowering characters was done.

\section{Vegetative growth characters}

Growth observations were measured at 30, 60, and 90, days after planting of corms. For getting the sharp results of following growth observations, four plants were selected in each plot mean values of the observations were expressed for representing of each treatment separately.

\section{Days of sprouting}

The days of sprouting of corms were recorded from the day after planting. The mean of the days of sprouting was obtain by some days of sprouting of four randomly selected plants and further average value was calculated.

\section{Plant height (cm)}

The height of the plant was measured from the soil surface to the tip of the longest leaves 
with the help of meter scale at 30,60 and 90 days after planting and their average was worked out.

\section{Number of leaves per plant}

The total number of leaves in four plants in each plot was counted at 30, 60, and 90 days after planting then average was worked out.

\section{Length of longest leaf $(\mathrm{cm})$}

The length of the longest leaf was measured with the help of meter scale of selected plants at 30, 60, and 90 days interval and average leaf length was calculated.

\section{Flowering characters}

The measure the flowering performance only those plants were observed in each plot, which were measured for the performance of vegetative growth. Here also mean values of the observations were expressed for representing of each treatment separately. The flowering observations recorded are as follows.

\section{Days required for visibility of first spike (Days)}

Days required for the visibility of first spike were counted from the date of planting of the corms.

Days required for opening of first floret (Days)

Days required for opening of first flower were counted from the date of planting of the corms.

\section{Number of florets per spike}

The numbers of florets per spike were counted and mean values were expressed.

\section{Diameter of floret $(\mathrm{cm})$}

The diameter of floret was measured with the help of vernier calipers and mean values were expressed in $\mathrm{cm}$.

\section{Diameter of spike (cm)}

Diameter of spike was measured with the help of Vernier calipers and mean values were expressed in $\mathrm{cm}$.

\section{Number of spikes per plant}

The number of the spikes per plant was counted and means values were expressed.

\section{Longevity of spike (Days)}

The longevity of spike was observed in the field. The data were recorded from first flower bud opening to last flower faded and mean values were expressed.

\section{Statistical analysis}

The experimental data were analyzed statistically by the techniques of analysis of variance described by Snedecor and Cochran (1967).

The significance of the treatments was tested with the help of "F" (variance ratio) test. Critical difference was calculated by the following formula.

$\mathrm{CD}$ at $5 \%=\frac{\sqrt{2 \mathrm{EMS}} X \mathrm{t}}{\mathrm{r}}$

Where, $\mathrm{CD}=$ Critical difference

EMS $=$ Error means sum of square

$\mathrm{r}=$ Number of replications

$\mathrm{t}=\mathrm{t}$ value at $5 \%$ level of significance at error degree of freedom. 


\section{Results and Discussion}

\section{Vegetative growth characters}

The data pertaining to the days of sprouting as presented in table 1 showed that the minimum days taken to sprouting of corms (10.28) were recorded under the treatments of $\mathrm{GA}_{3}$ at $800 \mathrm{ppm}$ while maximum days taken to sprouting of corms (14.67) were recorded under the control. Similar results with the application of $\mathrm{GA}_{3}$ have been also reported by Kirad et al., (2001), Sharma et al., (2006) and Kumar et al., (2009) in gladiolus.

Out of the growth regulating chemicals the maximum plant height $(95.53 \mathrm{~cm})$ was observed under the treatment GA3 at 400ppm applied as corm dipping alone whereas, the minimum plant height i.e. $(86.40 \mathrm{~cm})$ was found under the control. This observation on increase in the plant height with the application of $\mathrm{GA}_{3}$ are in agreement with the findings of Kirad et al., (2001), Prasad et al., (2002), Tawar et al., (2002) and Kumar et al., (2005) in gladiolus.

It is also clear from the data that maximum number of leaves per plant i.e. (5.66) were recorded under treatment of $\mathrm{GA}_{3}$ at $400 \mathrm{ppm}$ applied as corm dipping alone whereas, the minimum number of leaves per plant (5.19) was recorded under control. The findings are in conformity with the observation of Kirad $e t$ al., (2001), Kumar et al., (2005) in gladiolus.

It is evident from the data that the significantly maximum length of the leaf per plant (57.23 $\mathrm{cm}$ ) was recorded under the treatment $\mathrm{GA}_{3}$ at $800 \mathrm{ppm}$ applied as corm dipping + foliar spraying whereas, the minimum length of the leaf was recorded to be $(50.25 \mathrm{~cm})$ under control. Similar increases in the length of the leaf with application of $\mathrm{GA}_{3}$ have been also reported by Gaur et al., (2003), Rana et al., (2005) and Sharma et al., (2006) in gladiolus (Table 2-4).

\section{Flowering characters}

The data on days taken to the number of days required for the visibility of first spike from the date of planting of corms as presented in table 5 that the minimum days taken for visibility of first spike (86.54) were found under the treatment BA at 25ppm applied as foliar spraying followed and the maximum days taken for visibility of first spike (87.59) under the BA treatment at 100ppm applied as corm dipping + foliar spraying alone. Similar results on the visibility of first spike with the application of BA have been also reported by Mahesh and Misra (1993) in gladiolus. Significantly, minimum number of days taken to complete opening of first floret on the spike (89.71) noted under the $\mathrm{GA}_{3}$ treatment at 800ppm applied as corm dipping + foliar spraying alone.

However, the maximum number of days taken to complete opening of first floret on the spike from the date of planting were observed to be (100.81) under the control. An early flowering in gladiolus with the application of $\mathrm{GA}_{3}$ has been also reported by Ravidas et al., (1992), Kumar et al., (2008) and Chaudhray et al., (2018) in gladiolus (Table 6).

The data in the given table 7 clearly reveals that the maximum numbers of spikes per plant (1.080) were observed under the treatment $\mathrm{GA}_{3}$ at $400 \mathrm{ppm}$ applied as foliar spraying and the minimum number of spikes per plant (1.017) was recorded under treatment BA at $100 \mathrm{ppm}$ applied as corm dipping alone. 
Table.1 Effect of plant growth regulators on days taken to sprouting of corms in gladiolus cv. White Prosperity

\begin{tabular}{|c|c|c|}
\hline S. No. & Treatments & Days of sprouting \\
\hline \multirow[t]{2}{*}{1.} & Control & 14.67 \\
\hline & Corm dipping & \\
\hline 2. & $\mathrm{GA}_{3} 200 \mathrm{ppm}$ & 11.34 \\
\hline 3. & $\mathrm{GA}_{3} 400 \mathrm{ppm}$ & 11.33 \\
\hline 4. & $\mathrm{GA}_{3} 800 \mathrm{ppm}$ & 10.28 \\
\hline 5. & NAA 200 ppm & 10.40 \\
\hline 6. & NAA $400 \mathrm{ppm}$ & 10.78 \\
\hline 7. & NAA $600 \mathrm{ppm}$ & 11.43 \\
\hline 8. & BA $25 \mathrm{ppm}$ & 12.33 \\
\hline 9. & BA $50 \mathrm{ppm}$ & 12.46 \\
\hline \multirow[t]{2}{*}{10.} & BA $100 \mathrm{ppm}$ & 14.25 \\
\hline & Foliar spraying & \\
\hline 11. & $\mathrm{GA}_{3} 200 \mathrm{ppm}$ & 14.20 \\
\hline 12. & $\mathrm{GA}_{3} 400 \mathrm{ppm}$ & 14.25 \\
\hline 13. & $\mathrm{GA}_{3} 800 \mathrm{ppm}$ & 14.51 \\
\hline 14. & NAA $200 \mathrm{ppm}$ & 14.51 \\
\hline 15. & NAA $400 \mathrm{ppm}$ & 13.65 \\
\hline 16. & NAA $600 \mathrm{ppm}$ & 14.23 \\
\hline 17. & BA $25 \mathrm{ppm}$ & 14.20 \\
\hline 18. & BA $50 \mathrm{ppm}$ & 14.44 \\
\hline \multirow[t]{2}{*}{19.} & BA $100 \mathrm{ppm}$ & 13.45 \\
\hline & Corm dipping + foliar spraying & \\
\hline 20. & $\mathrm{GA}_{3} 200 \mathrm{ppm}$ & 11.35 \\
\hline 21. & $\mathrm{GA}_{3} 400 \mathrm{ppm}$ & 11.31 \\
\hline 22. & $\mathrm{GA}_{3} 800 \mathrm{ppm}$ & 10.23 \\
\hline 23. & NAA 200 ppm & 10.38 \\
\hline 24. & NAA 400 ppm & 10.77 \\
\hline 25. & NAA 600 ppm & 11.46 \\
\hline 26. & BA $25 \mathrm{ppm}$ & 12.46 \\
\hline 27. & BA $50 \mathrm{ppm}$ & 12.51 \\
\hline \multirow[t]{3}{*}{28.} & BA $100 \mathrm{ppm}$ & 14.22 \\
\hline & C.D. at 5\% & 0.17 \\
\hline & $\operatorname{SE}(m) \pm$ & 0.06 \\
\hline
\end{tabular}


Table.2 Effect of plant growth regulators on plant height $(\mathrm{cm})$ at different growth stages in gladiolus cv. White Prosperity

\begin{tabular}{|c|c|c|c|c|}
\hline \multirow[t]{2}{*}{ S. No. } & \multirow[t]{2}{*}{ Treatments } & \multicolumn{3}{|c|}{ Plant Height (cm) } \\
\hline & & 30 DAP & 60 DAP & 90 DAP \\
\hline \multirow[t]{2}{*}{1.} & Control & 40.40 & 60.61 & 86.40 \\
\hline & Corm dipping & & & \\
\hline 2. & $\mathrm{GA}_{3} 200 \mathrm{ppm}$ & 45.38 & 65.63 & 90.93 \\
\hline 3. & $\mathrm{GA}_{3} 400 \mathrm{ppm}$ & 45.55 & 67.26 & 95.53 \\
\hline 4. & $\mathrm{GA}_{3} 800 \mathrm{ppm}$ & 45.63 & 66.73 & 90.89 \\
\hline 5. & NAA $200 \mathrm{ppm}$ & 45.50 & 65.81 & 89.69 \\
\hline 6. & NAA $400 \mathrm{ppm}$ & 45.61 & 65.78 & 88.73 \\
\hline 7. & NAA $600 \mathrm{ppm}$ & 45.53 & 65.83 & 90.48 \\
\hline 8. & BA $25 \mathrm{ppm}$ & 45.63 & 68.84 & 90.83 \\
\hline 9. & BA $50 \mathrm{ppm}$ & 45.61 & 66.88 & 88.74 \\
\hline \multirow[t]{2}{*}{10.} & BA $100 \mathrm{ppm}$ & 45.66 & 66.80 & 88.90 \\
\hline & Foliar Spraying & & & \\
\hline 11. & $\mathrm{GA}_{3} 200 \mathrm{ppm}$ & 45.80 & 67.73 & 90.70 \\
\hline 12. & $\mathrm{GA}_{3} 400 \mathrm{ppm}$ & 45.20 & 65.63 & 89.58 \\
\hline 13. & $\mathrm{GA}_{3} 800 \mathrm{ppm}$ & 45.45 & 66.56 & 90.73 \\
\hline 14. & NAA $200 \mathrm{ppm}$ & 46.72 & 66.91 & 89.33 \\
\hline 15. & NAA 400 ppm & 45.90 & 67.13 & 88.17 \\
\hline 16. & NAA 600 ppm & 46.80 & 66.60 & 90.39 \\
\hline 17. & BA 25 ppm & 46.63 & 67.26 & 87.69 \\
\hline 18. & BA $50 \mathrm{ppm}$ & 46.73 & 67.36 & 86.77 \\
\hline \multirow[t]{2}{*}{19.} & BA $100 \mathrm{ppm}$ & 46.35 & 67.81 & 90.72 \\
\hline & Corm dipping + foliar spraying & & & \\
\hline 20. & $\mathrm{GA}_{3} 200 \mathrm{ppm}$ & 46.45 & 69.21 & 91.43 \\
\hline 21. & $\mathrm{GA}_{3} 400 \mathrm{ppm}$ & 46.53 & 69.30 & 90.39 \\
\hline 22. & $\mathrm{GA}_{3} 800 \mathrm{ppm}$ & 46.62 & 68.82 & 90.70 \\
\hline 23. & NAA $200 \mathrm{ppm}$ & 46.85 & 69.25 & 92.60 \\
\hline 24. & NAA $400 \mathrm{ppm}$ & 47.62 & 70.17 & 92.95 \\
\hline 25. & NAA $600 \mathrm{ppm}$ & 46.42 & 70.20 & 90.56 \\
\hline 26. & BA $25 \mathrm{ppm}$ & 47.84 & 70.33 & 90.83 \\
\hline 27. & BA $50 \mathrm{ppm}$ & 46.85 & 70.35 & 89.34 \\
\hline \multirow[t]{3}{*}{28.} & BA $100 \mathrm{ppm}$ & 46.90 & 70.43 & 90.65 \\
\hline & C.D. at 5\% & 0.12 & 0.22 & 1.13 \\
\hline & $\operatorname{SE}(m) \pm$ & 0.04 & 0.07 & 0.40 \\
\hline
\end{tabular}


Table.3 Effect of plant growth regulators on number of leaves per plant in gladiolus cv. White Prosperity

\begin{tabular}{|c|c|c|c|c|}
\hline \multirow[t]{2}{*}{ S. No. } & \multirow[t]{2}{*}{ Treatments } & \multicolumn{3}{|c|}{ Number of leaves per plant } \\
\hline & & 30 DAP & 60 DAP & 90 DAP \\
\hline \multirow[t]{2}{*}{1.} & Control & 2.33 & 4.13 & 5.19 \\
\hline & Corm dipping & & & \\
\hline 2. & $\mathrm{GA}_{3} 200 \mathrm{ppm}$ & 2.67 & 4.52 & 5.47 \\
\hline 3. & $\mathrm{GA}_{3} 400 \mathrm{ppm}$ & 2.88 & 4.54 & 5.66 \\
\hline 4. & $\mathrm{GA}_{3} 800 \mathrm{ppm}$ & 2.96 & 4.56 & 5.37 \\
\hline 5. & NAA 200 ppm & 2.64 & 4.56 & 5.30 \\
\hline 6. & NAA 400 ppm & 2.67 & 4.90 & 5.62 \\
\hline 7. & NAA 600 ppm & 2.68 & 4.96 & 5.38 \\
\hline 8. & BA 25 ppm & 2.85 & 4.92 & 5.41 \\
\hline 9. & BA 50 ppm & 2.94 & 4.91 & 5.30 \\
\hline \multirow[t]{2}{*}{10.} & BA 100 ppm & 2.97 & 4.95 & 5.43 \\
\hline & Foliar Spraying & & & \\
\hline 11. & $\mathrm{GA}_{3} 200 \mathrm{ppm}$ & 2.91 & 4.62 & 5.36 \\
\hline 12. & $\mathrm{GA}_{3} 400 \mathrm{ppm}$ & 2.93 & 4.65 & 5.38 \\
\hline 13. & $\mathrm{GA}_{3} 800 \mathrm{ppm}$ & 2.95 & 4.67 & 5.62 \\
\hline 14. & NAA $200 \mathrm{ppm}$ & 2.95 & 4.93 & 5.60 \\
\hline 15. & NAA 400 ppm & 2.97 & 4.96 & 5.39 \\
\hline 16. & NAA $600 \mathrm{ppm}$ & 2.97 & 4.98 & 5.37 \\
\hline 17. & BA 25 ppm & 2.85 & 4.95 & 5.25 \\
\hline 18. & BA 50 ppm & 2.92 & 4.95 & 5.24 \\
\hline \multirow[t]{2}{*}{19.} & BA 100 ppm & 2.95 & 4.96 & 5.34 \\
\hline & Corm dipping + foliar spraying & & & \\
\hline 20. & $\mathrm{GA}_{3} 200 \mathrm{ppm}$ & 2.96 & 4.55 & 5.40 \\
\hline 21. & $\mathrm{GA}_{3} 400 \mathrm{ppm}$ & 2.98 & 4.60 & 5.44 \\
\hline 22. & $\mathrm{GA}_{3} 800 \mathrm{ppm}$ & 2.95 & 4.63 & 5.30 \\
\hline 23. & NAA 200 ppm & 2.87 & 4.50 & 5.40 \\
\hline 24. & NAA 400 ppm & 2.90 & 4.53 & 5.50 \\
\hline 25. & NAA 600 ppm & 2.93 & 4.59 & 5.56 \\
\hline 26. & BA 25 ppm & 2.97 & 4.30 & 5.34 \\
\hline 27. & BA 50 ppm & 2.98 & 4.35 & 5.64 \\
\hline \multirow[t]{3}{*}{28.} & BA 100 ppm & 2.97 & 4.36 & 5.36 \\
\hline & C.D. at $5 \%$ & 0.026 & 0.047 & 0.041 \\
\hline & $\mathrm{SE}(\mathrm{m}) \pm$ & 0.009 & 0.016 & 0.015 \\
\hline
\end{tabular}


Table.4 Effect of plant growth regulators on length of longest leaf $(\mathrm{cm})$ in gladiolus $\mathrm{cv}$. White Prosperity

\begin{tabular}{|c|c|c|c|c|}
\hline \multirow[t]{2}{*}{ S. No. } & \multirow[t]{2}{*}{ Treatments } & \multicolumn{3}{|c|}{ Length of longest leaf (cm) } \\
\hline & & 30 DAP & 60 DAP & 90 DAP \\
\hline \multirow[t]{2}{*}{1.} & Control & 25.17 & 43.40 & 50.25 \\
\hline & Corm dipping & & & \\
\hline 2. & $\mathrm{GA}_{3} 200 \mathrm{ppm}$ & 26.32 & 44.92 & 54.37 \\
\hline 3. & $\mathrm{GA}_{3} 400 \mathrm{ppm}$ & 26.38 & 44.81 & 55.79 \\
\hline 4. & $\mathrm{GA}_{3} 800 \mathrm{ppm}$ & 26.39 & 45.25 & 56.65 \\
\hline 5. & NAA $200 \mathrm{ppm}$ & 25.73 & 44.28 & 53.38 \\
\hline 6. & NAA 400 ppm & 25.79 & 44.59 & 55.33 \\
\hline 7. & NAA $600 \mathrm{ppm}$ & 25.81 & 44.63 & 55.20 \\
\hline 8. & BA 25 ppm & 25.84 & 44.89 & 53.72 \\
\hline 9. & BA 50 ppm & 25.87 & 44.92 & 55.42 \\
\hline \multirow[t]{2}{*}{10.} & BA 100 ppm & 25.92 & 44.95 & 53.73 \\
\hline & Foliar spraying & & & \\
\hline 11. & $\mathrm{GA}_{3} 200 \mathrm{ppm}$ & 26.17 & 44.92 & 54.45 \\
\hline 12. & $\mathrm{GA}_{3} 400 \mathrm{ppm}$ & 25.91 & 45.86 & 55.93 \\
\hline 13. & $\mathrm{GA}_{3} 800 \mathrm{ppm}$ & 26.15 & 45.93 & 56.83 \\
\hline 14. & NAA $200 \mathrm{ppm}$ & 26.41 & 44.85 & 53.45 \\
\hline 15. & NAA 400 ppm & 26.50 & 45.95 & 55.95 \\
\hline 16. & NAA $600 \mathrm{ppm}$ & 27.20 & 45.61 & 54.18 \\
\hline 17. & BA 25 ppm & 27.26 & 45.40 & 54.45 \\
\hline 18. & BA $50 \mathrm{ppm}$ & 27.26 & 45.36 & 54.86 \\
\hline \multirow[t]{2}{*}{19.} & BA $100 \mathrm{ppm}$ & 26.83 & 44.93 & 54.71 \\
\hline & Corm dipping + foliar spraying & & & \\
\hline 20. & $\mathrm{GA}_{3} 200 \mathrm{ppm}$ & 26.13 & 45.46 & 55.20 \\
\hline 21. & $\mathrm{GA}_{3} 400 \mathrm{ppm}$ & 27.16 & 45.66 & 56.20 \\
\hline 22. & $\mathrm{GA}_{3} 800 \mathrm{ppm}$ & 27.15 & 45.76 & 57.23 \\
\hline 23. & NAA 200 ppm & 25.73 & 44.93 & 55.52 \\
\hline 24. & NAA $400 \mathrm{ppm}$ & 25.84 & 45.21 & 55.93 \\
\hline 25. & NAA 600 ppm & 25.95 & 45.38 & 54.43 \\
\hline 26. & BA 25 ppm & 25.51 & 44.80 & 51.87 \\
\hline 27. & BA 50 ppm & 25.89 & 44.85 & 55.91 \\
\hline \multirow[t]{3}{*}{28.} & BA 100 ppm & 25.81 & 44.96 & 51.77 \\
\hline & C.D. at 5\% & 0.06 & 0.17 & 3.18 \\
\hline & $\mathrm{SE}(\mathrm{m}) \pm$ & 0.02 & 0.06 & 1.12 \\
\hline
\end{tabular}


Table.5 Effect of plant growth regulators on days required for visibility of first spike in gladiolus cv. White Prosperity

\begin{tabular}{|c|c|c|}
\hline S. No. & Treatments & $\begin{array}{l}\text { Days required for visibility of } \\
\text { first spike (DAP) }\end{array}$ \\
\hline \multirow[t]{2}{*}{1.} & Control & 87.93 \\
\hline & Corm dipping & \\
\hline 2. & $\mathrm{GA}_{3} 200 \mathrm{ppm}$ & 83.57 \\
\hline 3. & $\mathrm{GA}_{3} 400 \mathrm{ppm}$ & 82.40 \\
\hline 4. & $\mathrm{GA}_{3} 800 \mathrm{ppm}$ & 81.73 \\
\hline 5. & NAA $200 \mathrm{ppm}$ & 86.26 \\
\hline 6. & NAA 400 ppm & 86.45 \\
\hline 7. & NAA 600 ppm & 86.65 \\
\hline 8. & BA $25 \mathrm{ppm}$ & 86.75 \\
\hline 9. & BA $50 \mathrm{ppm}$ & 86.57 \\
\hline \multirow[t]{2}{*}{10.} & BA $100 \mathrm{ppm}$ & 86.84 \\
\hline & Foliar spraying & \\
\hline 11. & $\mathrm{GA}_{3} 200 \mathrm{ppm}$ & 84.48 \\
\hline 12. & $\mathrm{GA}_{3} 400 \mathrm{ppm}$ & 84.32 \\
\hline 13. & $\mathrm{GA}_{3} 800 \mathrm{ppm}$ & 84.91 \\
\hline 14. & NAA 200 ppm & 86.64 \\
\hline 15. & NAA $400 \mathrm{ppm}$ & 86.13 \\
\hline 16. & NAA $600 \mathrm{ppm}$ & 86.61 \\
\hline 17. & BA $25 \mathrm{ppm}$ & 86.54 \\
\hline 18. & BA $50 \mathrm{ppm}$ & 86.67 \\
\hline \multirow[t]{2}{*}{19.} & BA $100 \mathrm{ppm}$ & 86.86 \\
\hline & Corm dipping + foliar spraying & \\
\hline 20. & $\mathrm{GA}_{3} 200 \mathrm{ppm}$ & 81.34 \\
\hline 21. & $\mathrm{GA}_{3} 400 \mathrm{ppm}$ & 80.70 \\
\hline 22. & $\mathrm{GA}_{3} 800 \mathrm{ppm}$ & 78.84 \\
\hline 23. & NAA $200 \mathrm{ppm}$ & 85.92 \\
\hline 24. & NAA $400 \mathrm{ppm}$ & 87.10 \\
\hline 25. & NAA $600 \mathrm{ppm}$ & 87.27 \\
\hline 26. & BA $25 \mathrm{ppm}$ & 87.34 \\
\hline 27. & BA $50 \mathrm{ppm}$ & 87.33 \\
\hline \multirow[t]{3}{*}{28.} & BA $100 \mathrm{ppm}$ & 87.59 \\
\hline & C.D. at 5\% & 0.40 \\
\hline & $\mathrm{SE}(\mathbf{m}) \pm$ & 0.14 \\
\hline
\end{tabular}


Table.6 Effect of plant growth regulators on days required for opening of first floret in gladiolus cv. White Prosperity

\begin{tabular}{|c|c|c|}
\hline S. No. & Treatments & $\begin{array}{l}\text { Days required for opening of } \\
\text { first floret (DAP) }\end{array}$ \\
\hline \multirow[t]{2}{*}{1.} & Control & 100.81 \\
\hline & Corm dipping & \\
\hline 2. & $\mathrm{GA}_{3} 200 \mathrm{ppm}$ & 98.37 \\
\hline 3. & $\mathrm{GA}_{3} 400 \mathrm{ppm}$ & 97.47 \\
\hline 4. & $\mathrm{GA}_{3} 800 \mathrm{ppm}$ & 90.67 \\
\hline 5. & NAA 200 ppm & 99.45 \\
\hline 6. & NAA $400 \mathrm{ppm}$ & 99.34 \\
\hline 7. & NAA $600 \mathrm{ppm}$ & 99.61 \\
\hline 8. & BA $25 \mathrm{ppm}$ & 99.75 \\
\hline 9. & BA $50 \mathrm{ppm}$ & 100.06 \\
\hline \multirow[t]{2}{*}{10.} & BA $100 \mathrm{ppm}$ & 100.17 \\
\hline & Foliar spraying & \\
\hline 11. & $\mathrm{GA}_{3} 200 \mathrm{ppm}$ & 96.55 \\
\hline 12. & $\mathrm{GA}_{3} 400 \mathrm{ppm}$ & 96.28 \\
\hline 13. & $\mathrm{GA}_{3} 800 \mathrm{ppm}$ & 96.81 \\
\hline 14. & NAA 200 ppm & 100.16 \\
\hline 15. & NAA 400 ppm & 99.62 \\
\hline 16. & NAA 600 ppm & 99.87 \\
\hline 17. & BA $25 \mathrm{ppm}$ & 99.16 \\
\hline 18. & BA $50 \mathrm{ppm}$ & 99.28 \\
\hline \multirow[t]{2}{*}{19.} & BA $100 \mathrm{ppm}$ & 100.12 \\
\hline & Corm dipping + foliar spraying & \\
\hline 20. & $\mathrm{GA}_{3} 200 \mathrm{ppm}$ & 96.56 \\
\hline 21. & $\mathrm{GA}_{3} 400 \mathrm{ppm}$ & 90.65 \\
\hline 22. & $\mathrm{GA}_{3} 800 \mathrm{ppm}$ & 89.71 \\
\hline 23. & NAA 200 ppm & 99.41 \\
\hline 24. & NAA $400 \mathrm{ppm}$ & 100.16 \\
\hline 25. & NAA 600 ppm & 100.05 \\
\hline 26. & BA $25 \mathrm{ppm}$ & 100.03 \\
\hline 27. & BA $50 \mathrm{ppm}$ & 99.91 \\
\hline \multirow[t]{3}{*}{28.} & BA $100 \mathrm{ppm}$ & 99.95 \\
\hline & C.D. at $5 \%$ & 0.34 \\
\hline & $\mathrm{SE}(\mathrm{m}) \pm$ & 0.12 \\
\hline
\end{tabular}


Table.7 Effect of plant growth regulators on number of spike per plant in gladiolus cv. White Prosperity

\begin{tabular}{|c|c|c|}
\hline S. No. & Treatments & Number of spike per plant \\
\hline \multirow[t]{2}{*}{1.} & Control & 1.000 \\
\hline & Corm dipping & \\
\hline 2. & $\mathrm{GA}_{3} 200 \mathrm{ppm}$ & 1.023 \\
\hline 3. & $\mathrm{GA}_{3} 400 \mathrm{ppm}$ & 1.033 \\
\hline 4. & $\mathrm{GA}_{3} 800 \mathrm{ppm}$ & 1.043 \\
\hline 5. & NAA $200 \mathrm{ppm}$ & 1.040 \\
\hline 6. & NAA $400 \mathrm{ppm}$ & 1.047 \\
\hline 7. & NAA $600 \mathrm{ppm}$ & 1.043 \\
\hline 8. & BA $25 \mathrm{ppm}$ & 1.053 \\
\hline 9. & BA $50 \mathrm{ppm}$ & 1.060 \\
\hline \multirow[t]{2}{*}{10.} & BA $100 \mathrm{ppm}$ & 1.017 \\
\hline & Foliar spraying & \\
\hline 11. & $\mathrm{GA}_{3} 200 \mathrm{ppm}$ & 1.060 \\
\hline 12. & $\mathrm{GA}_{3} 400 \mathrm{ppm}$ & 1.080 \\
\hline 13. & $\mathrm{GA}_{3} 800 \mathrm{ppm}$ & 1.070 \\
\hline 14. & NAA 200 ppm & 1.053 \\
\hline 15. & NAA 400 ppm & 1.063 \\
\hline 16. & NAA 600 ppm & 1.067 \\
\hline 17. & BA $25 \mathrm{ppm}$ & 1.030 \\
\hline 18. & BA $50 \mathrm{ppm}$ & 1.040 \\
\hline \multirow[t]{2}{*}{19.} & BA $100 \mathrm{ppm}$ & 1.050 \\
\hline & Corm dipping + foliar spraying & \\
\hline 20. & $\mathrm{GA}_{3} 200 \mathrm{ppm}$ & 1.053 \\
\hline 21. & $\mathrm{GA}_{3} 400 \mathrm{ppm}$ & 1.013 \\
\hline 22. & $\mathrm{GA}_{3} 800 \mathrm{ppm}$ & 1.067 \\
\hline 23. & NAA 200 ppm & 1.063 \\
\hline 24. & NAA 400 ppm & 1.070 \\
\hline 25. & NAA 600 ppm & 1.047 \\
\hline 26. & BA 25 ppm & 1.050 \\
\hline 27. & BA $50 \mathrm{ppm}$ & 1.053 \\
\hline \multirow[t]{3}{*}{28.} & BA $100 \mathrm{ppm}$ & 1.050 \\
\hline & C.D. at $5 \%$ & 0.029 \\
\hline & $\mathrm{SE}(\mathbf{m}) \pm$ & 0.010 \\
\hline
\end{tabular}


Table.8 Effect of plant growth regulators on number of floret per spike in gladiolus cv. White Prosperity

\begin{tabular}{|c|c|c|}
\hline S. No. & Treatments & Number of floret per spike \\
\hline \multirow[t]{2}{*}{1.} & Control & 10.72 \\
\hline & Corm dipping & \\
\hline 2. & $\mathrm{GA}_{3} 200 \mathrm{ppm}$ & 12.71 \\
\hline 3. & $\mathrm{GA}_{3} 400 \mathrm{ppm}$ & 13.27 \\
\hline 4. & $\mathrm{GA}_{3} 800 \mathrm{ppm}$ & 14.27 \\
\hline 5. & NAA 200 ppm & 11.20 \\
\hline 6. & NAA $400 \mathrm{ppm}$ & 11.75 \\
\hline 7. & NAA $600 \mathrm{ppm}$ & 11.81 \\
\hline 8. & BA $25 \mathrm{ppm}$ & 10.94 \\
\hline 9. & BA $50 \mathrm{ppm}$ & 10.83 \\
\hline \multirow[t]{2}{*}{10.} & BA $100 \mathrm{ppm}$ & 10.85 \\
\hline & Foliar spraying & \\
\hline 11. & $\mathrm{GA}_{3} 200 \mathrm{ppm}$ & 11.51 \\
\hline 12. & $\mathrm{GA}_{3} 400 \mathrm{ppm}$ & 12.66 \\
\hline 13. & $\mathrm{GA}_{3} 800 \mathrm{ppm}$ & 13.65 \\
\hline 14. & NAA 200 ppm & 11.33 \\
\hline 15. & NAA $400 \mathrm{ppm}$ & 11.30 \\
\hline 16. & NAA $600 \mathrm{ppm}$ & 11.48 \\
\hline 17. & BA 25 ppm & 10.83 \\
\hline 18. & BA $50 \mathrm{ppm}$ & 10.88 \\
\hline \multirow[t]{2}{*}{19.} & BA $100 \mathrm{ppm}$ & 10.92 \\
\hline & Corm dipping + foliar spraying & \\
\hline 20. & $\mathrm{GA}_{3} 200 \mathrm{ppm}$ & 11.37 \\
\hline 21. & $\mathrm{GA}_{3} 400 \mathrm{ppm}$ & 12.49 \\
\hline 22. & $\mathrm{GA}_{3} 800 \mathrm{ppm}$ & 11.34 \\
\hline 23. & NAA $200 \mathrm{ppm}$ & 11.75 \\
\hline 24. & NAA $400 \mathrm{ppm}$ & 11.73 \\
\hline 25. & NAA $600 \mathrm{ppm}$ & 11.83 \\
\hline 26. & BA $25 \mathrm{ppm}$ & 10.75 \\
\hline 27. & BA $50 \mathrm{ppm}$ & 10.82 \\
\hline \multirow[t]{3}{*}{28.} & BA $100 \mathrm{ppm}$ & 10.84 \\
\hline & C.D. at 5\% & 0.15 \\
\hline & $\mathrm{SE}(\mathrm{m}) \pm$ & 0.05 \\
\hline
\end{tabular}


Table.9 Effect of plant growth regulators on diameter of floret $(\mathrm{cm})$ in gladiolus $\mathrm{cv}$. White Prosperity

\begin{tabular}{|c|c|c|}
\hline S. No. & Treatments & Diameter of floret $(\mathrm{cm})$ \\
\hline \multirow[t]{2}{*}{1.} & Control & 11.25 \\
\hline & Corm dipping & \\
\hline 2. & $\mathrm{GA}_{3} 200 \mathrm{ppm}$ & 11.86 \\
\hline 3. & $\mathrm{GA}_{3} 400 \mathrm{ppm}$ & 12.82 \\
\hline 4. & $\mathrm{GA}_{3} 800 \mathrm{ppm}$ & 11.54 \\
\hline 5. & NAA 200 ppm & 11.41 \\
\hline 6. & NAA $400 \mathrm{ppm}$ & 11.45 \\
\hline 7. & NAA $600 \mathrm{ppm}$ & 11.31 \\
\hline 8. & BA $25 \mathrm{ppm}$ & 11.32 \\
\hline 9. & BA $50 \mathrm{ppm}$ & 11.37 \\
\hline \multirow[t]{2}{*}{10.} & BA $100 \mathrm{ppm}$ & 11.48 \\
\hline & Foliar spraying & \\
\hline 11. & $\mathrm{GA}_{3} 200 \mathrm{ppm}$ & 11.67 \\
\hline 12. & $\mathrm{GA}_{3} 400 \mathrm{ppm}$ & 11.37 \\
\hline 13. & $\mathrm{GA}_{3} 800 \mathrm{ppm}$ & 11.54 \\
\hline 14. & NAA 200 ppm & 11.60 \\
\hline 15. & NAA $400 \mathrm{ppm}$ & 11.65 \\
\hline 16. & NAA $600 \mathrm{ppm}$ & 11.58 \\
\hline 17. & BA $25 \mathrm{ppm}$ & 11.25 \\
\hline 18. & BA $50 \mathrm{ppm}$ & 11.37 \\
\hline \multirow[t]{2}{*}{19.} & BA $100 \mathrm{ppm}$ & 11.39 \\
\hline & Corm dipping + foliar spraying & \\
\hline 20. & $\mathrm{GA}_{3} 200 \mathrm{ppm}$ & 11.61 \\
\hline 21. & $\mathrm{GA}_{3} 400 \mathrm{ppm}$ & 11.78 \\
\hline 22. & $\mathrm{GA}_{3} 800 \mathrm{ppm}$ & 11.42 \\
\hline 23. & NAA 200 ppm & 11.33 \\
\hline 24. & NAA 400 ppm & 11.40 \\
\hline 25. & NAA $600 \mathrm{ppm}$ & 11.36 \\
\hline 26. & BA $25 \mathrm{ppm}$ & 11.37 \\
\hline 27. & BA $50 \mathrm{ppm}$ & 11.41 \\
\hline \multirow[t]{3}{*}{28.} & BA $100 \mathrm{ppm}$ & 11.51 \\
\hline & C.D. at $5 \%$ & 0.13 \\
\hline & $\mathrm{SE}(\mathbf{m}) \pm$ & 0.04 \\
\hline
\end{tabular}


Table.10 Effect of plant growth regulators on diameter of spike $(\mathrm{cm})$ in gladiolus cv. White Prosperity

\begin{tabular}{|c|c|c|}
\hline S. No. & Treatments & Diameter of spike (cm) \\
\hline \multirow[t]{2}{*}{1.} & Control & 0.800 \\
\hline & Corm dipping & \\
\hline 2. & $\mathrm{GA}_{3} 200 \mathrm{ppm}$ & 0.867 \\
\hline 3. & $\mathrm{GA}_{3} 400 \mathrm{ppm}$ & 0.887 \\
\hline 4. & $\mathrm{GA}_{3} 800 \mathrm{ppm}$ & 0.837 \\
\hline 5. & NAA 200 ppm & 0.827 \\
\hline 6. & NAA $400 \mathrm{ppm}$ & 0.853 \\
\hline 7. & NAA $600 \mathrm{ppm}$ & 0.867 \\
\hline 8. & BA $25 \mathrm{ppm}$ & 0.883 \\
\hline 9. & BA $50 \mathrm{ppm}$ & 0.837 \\
\hline \multirow[t]{2}{*}{10.} & BA $100 \mathrm{ppm}$ & 0.853 \\
\hline & Foliar spraying & \\
\hline 11. & $\mathrm{GA}_{3} 200 \mathrm{ppm}$ & 0.853 \\
\hline 12. & $\mathrm{GA}_{3} 400 \mathrm{ppm}$ & 0.863 \\
\hline 13. & $\mathrm{GA}_{3} 800 \mathrm{ppm}$ & 0.837 \\
\hline 14. & NAA 200 ppm & 0.863 \\
\hline 15. & NAA $400 \mathrm{ppm}$ & 0.880 \\
\hline 16. & NAA $600 \mathrm{ppm}$ & 0.870 \\
\hline 17. & BA $25 \mathrm{ppm}$ & 0.870 \\
\hline 18. & BA $50 \mathrm{ppm}$ & 0.857 \\
\hline \multirow[t]{2}{*}{19.} & BA 100ppm & 0.850 \\
\hline & Corm dipping + foliar spraying & \\
\hline 20. & $\mathrm{GA}_{3} 200 \mathrm{ppm}$ & 0.870 \\
\hline 21. & $\mathrm{GA}_{3} 400 \mathrm{ppm}$ & 0.867 \\
\hline 22. & $\mathrm{GA}_{3} 800 \mathrm{ppm}$ & 0.843 \\
\hline 23. & NAA 200 ppm & 0.847 \\
\hline 24. & NAA 400 ppm & 0.850 \\
\hline 25. & NAA $600 \mathrm{ppm}$ & 0.873 \\
\hline 26. & BA $25 \mathrm{ppm}$ & 0.853 \\
\hline 27. & BA $50 \mathrm{ppm}$ & 0.837 \\
\hline \multirow[t]{3}{*}{28.} & BA $100 \mathrm{ppm}$ & 0.843 \\
\hline & C.D. at $5 \%$ & 0.029 \\
\hline & $\mathrm{SE}(\mathbf{m}) \pm$ & 0.010 \\
\hline
\end{tabular}


Table.11 Effect of plant growth regulators on longevity of spike (Days) in gladiolus cv. White Prosperity

\begin{tabular}{|c|c|c|}
\hline S. No. & Treatments & Longevity of spike (Days) \\
\hline \multirow[t]{2}{*}{1.} & Control & 12.95 \\
\hline & Corm dipping & \\
\hline 2. & $\mathrm{GA}_{3} 200 \mathrm{ppm}$ & 15.49 \\
\hline 3. & $\mathrm{GA}_{3} 400 \mathrm{ppm}$ & 16.81 \\
\hline 4. & $\mathrm{GA}_{3} 800 \mathrm{ppm}$ & 17.48 \\
\hline 5. & NAA 200 ppm & 13.59 \\
\hline 6. & NAA $400 \mathrm{ppm}$ & 14.26 \\
\hline 7. & NAA $600 \mathrm{ppm}$ & 14.27 \\
\hline 8. & BA $25 \mathrm{ppm}$ & 14.44 \\
\hline 9. & BA $50 \mathrm{ppm}$ & 15.44 \\
\hline \multirow[t]{2}{*}{10.} & BA $100 \mathrm{ppm}$ & 15.52 \\
\hline & Foliar spraying & \\
\hline 11. & $\mathrm{GA}_{3} 200 \mathrm{ppm}$ & 15.25 \\
\hline 12. & $\mathrm{GA}_{3} 400 \mathrm{ppm}$ & 16.38 \\
\hline 13. & $\mathrm{GA}_{3} 800 \mathrm{ppm}$ & 15.87 \\
\hline 14. & NAA 200 ppm & 13.92 \\
\hline 15. & NAA 400 ppm & 14.71 \\
\hline 16. & NAA 600 ppm & 15.86 \\
\hline 17. & BA $25 \mathrm{ppm}$ & 14.95 \\
\hline 18. & BA $50 \mathrm{ppm}$ & 15.36 \\
\hline \multirow[t]{2}{*}{19.} & BA $100 \mathrm{ppm}$ & 15.45 \\
\hline & Corm dipping + foliar spraying & \\
\hline 20. & $\mathrm{GA}_{3} 200 \mathrm{ppm}$ & 14.92 \\
\hline 21. & $\mathrm{GA}_{3} 400 \mathrm{ppm}$ & 15.81 \\
\hline 22. & $\mathrm{GA}_{3} 800 \mathrm{ppm}$ & 16.37 \\
\hline 23. & NAA 200 ppm & 13.69 \\
\hline 24. & NAA 400 ppm & 14.72 \\
\hline 25. & NAA 600 ppm & 15.49 \\
\hline 26. & BA $25 \mathrm{ppm}$ & 15.11 \\
\hline 27. & BA $50 \mathrm{ppm}$ & 15.44 \\
\hline \multirow[t]{3}{*}{28.} & BA $100 \mathrm{ppm}$ & 15.52 \\
\hline & C.D. at $5 \%$ & 0.25 \\
\hline & $\mathrm{SE}(\mathbf{m}) \pm$ & 0.08 \\
\hline
\end{tabular}

Similar results of alemaximum number of spikes with foliar spraying has been also reported by Chopde et al., (2013) and Yadav and Bhatia (2018) in gladiolus. The data on the number of florets per spike as presented in table 8 showed that the maximum number of florets per spike
(14.27) was observed under the treatment $\mathrm{GA}_{3}$ at 800ppm applied as corm dipping while the minimum number of spikes per plant (1.00) was obtained under the control. Similar increases in the number of florets with the application of $\mathrm{GA}_{3}$ have been also reported by Prasad et al., 
(2002), Gaur et al., (2003), Kumar and Singh (2005) and Chaudhray et al., (2018) in gladiolus.

The diameter of florets $(11.25 \mathrm{~cm})$ was noted significantly lower $(11.86 \mathrm{~cm})$ under control. Out of growth regulators treatments, the significantly greater diameter of florets $(12.82 \mathrm{~cm})$ was observed under the treatments $\mathrm{GA}_{3}$ at 400ppm applied as corm dipping alone. These results are in close conformity with the findings of Ram et al., (2001), Aier et al., (2015) and Chaudhray et al., (2018) in gladiolus (Table 9).

It is evident from the data that the diameter of spike $(0.800 \mathrm{~cm})$ was registered minimum under the control while the maximum diameter of the spike $(0.887 \mathrm{~cm})$ was found under the treatment $\mathrm{GA}_{3}$ at $400 \mathrm{ppm}$ concentration applied as corm dipping alone The similar results have been also reported by Attia et al., (2001) and Chopde et al., (2013) in gladiolus (Table 10).

The longevity of (12.95 days) was recorded under control. Out of the growth regulating chemicals (Table 11), the significantly maximum longevity of spike (17.48 days) was obtained under the treatment of $\mathrm{GA}_{3}$ at $800 \mathrm{ppm}$ applied as corm dipping alone. The favorable effects of $\mathrm{GA}_{3}$ application in promoting the longevity of whole spike have also been reported by Ram et al., (2001), Gaur et al., (2003) and Kumar and Singh (2005) in gladiolus.

On the basis of above finding, it can be concluded that $\mathrm{GA}_{3}$ at $400 \mathrm{ppm}$ or $800 \mathrm{ppm}$ concentration was significantly superior to other treatments in improving the growth, days of sprouting, plant height, length of longest leaf, number of days required for visibility of first spike, days required for opening of first floret, diameter of spike, number of florets per spike, diameter of floret, longevity of spike. NAA at 200, 400 and 600 ppm increases the number of leaves per plant, plant height. When the different treatments of application were compared, it was found that corm dipping + foliar spraying treatment was significantly superior to other treatments.

\section{References}

Aier, S., Langthasa, S., Hazarika, D. N., Gautam, B. P. and Goswami, R. K. (2015). Influence of $\mathrm{GA}_{3}$ and $\mathrm{BA}$ on morphological, phonological and yield attributes in gladiolus cv. Red Candyman. Journal of Agriculture and Veterinary Science, 8(6): 37-42.

Attia, F. A. (2001). The response of gladiolus (Gladiolus hybridus Hort.) cv. Rose Supreme, to different growth substances with different methods of application. Annals of Agricultural Science, 39(3): 169-171.

Chaudhary, P., Moond, S. K. and Bola, P. (2018). Effect of bioregulators on vegetative growth and flower production of gladiolus (Gladiolus hybridus Hort.). International Journal of Current Microbial Applied Science, 7: 463-470.

Chopde, N., Gonge, V. S. and Warade, A. D. (2013). Influence of growth regulators on gladiolus varieties. Journal of Agriculture Research and Technology, 38(3): 369-374.

Gaur, G. S. Chaudhary, T. C. and Trivedi, J. D. (2003). Effect of $\mathrm{GA}_{3}$ and IAA on growth, flowering and corm production in gladiolus cv. Eurovision. Journal of Farm Science, 12(1): 1-3.

Jenkins, J. M., Milholland, R. D., Lilly, J. P. and Beute, M. K. (1970). Commercial gladiolus production in North Carline. North Carolina Agriculture Extension Circle, 44: 1-34.

Kirad, K. S., Banafar, R. N. S., Barche, S., Billore, M. and Meenakshi, D. (2001). Effect of growth regulators on gladiolus. Annals of Agricultural Research, 22(2): 278-281.

Kumar, P. S., Bhagawati, R., Kumar, R. and Ronya, T. (2008). Effect of plant growth regulators on vegetative growth, flowering and corm production of 
gladiolus in Arunachal Pradesh. Journal of Ornamental Horticulture, 11(4): 265270.

Kumar, S. K., Chandrashekar, R., Padma, M. and Shivshankar, A. (2009). Effect of plant growth regulators on dormancy, corm and cormel production in gladiolus. Journal of Ornamental Horticulture, 12: 182-187.

Kumar, V. and Singh, R. P. (2005). Effect of soaking of mother corms with plant growth regulators on vegetative growth, flowering and corm production in gladiolus. Journal of Ornamental Horticulture, 8(4): 306-308.

Lepcha, B., Nautiyal, M. C. and Rao, V. K. (2007). Variability studies in gladiolus under mid hill conditions of Uttarakhand. Journal of Ornamental Horticulture, 10(1): 169-172.

Mahesh, K. S. and Misra, R. L. (1993). Effect of growth regulators on gladiolus. Journal of Ornamental Horticulture, 1(2): 12-15.

Nuvale, M. U., Aklade, S. A., Desai J. R. and Nannvare, P. V. (2010). Influence of plant growth regulators on growth, flowering and yield of chrysanthemum cv. 'IIHR-6. International Journal of Pharma Bio Science, 1: 1-4.

Olszewski, N., Sun, T. P. and Gubler, F. (2002). Gibberellins signaling: Biosynthesis, catabolism and response pathways. Plant Cell, 14: 61-80.

Padmalatha, T., Reddy, G. S., Chandrasekhar, R., Shankar, A. S. and Chaturvedi, A. (2013). Effect of pre-planting soaking of corms with chemicals and plant growth regulators on dormancy breaking and corm and cormel production in gladiolus. International Journal of Plant, Animal and Environmental
Science, 3(1): 28-33.

Prasad, A., Kumar, R., Arya, S. and Saxena, K. (2002). Varietal response of gladiolus corms to $\mathrm{GA}_{3}$ dipping. Journal of Ornamental Horticulture, 5(1): 69-70.

Ram, D., Verma, J. P. and Verma, H. K. (2001). Effect of plant growth regulators on vegetative growth of gladiolus. Annals of Agriculture Biology Research, 6(1): 81-84.

Rana, P., Kumar, J. and Kumar, M. (2005). Response of $\mathrm{GA}_{3}$, plant spacing and planting depth on growth, flowering and corm production in gladiolus. Journal of Ornamental Horticulture, 8(1): 41-44.

Ravidas, L., Rajeevan, P. K. and Valsalakumari, P. K. (1992). Effect of foliar application of growth regulators on the growth, flowering and corm yield of gladiolus cv. Friendship. South Indian Horticulture, 40(6): 329-335.

Sharma, D. P., Chattar, Y. K. and Nishith, G. (2006). Effect of gibberellic acid on growth, flowering and corm yield in three cultivars of gladiolus. Journal of Ornamental Horticulture, 9(2): 106-109.

Snedecor, G. W. and Cochran, W. G. (1967). Statistical Methods. The Lowa State University Press, 308-310.

Tawar, R. V., Sable, A. S. and Giri, M. D. (2002). Effect of growth regulators on growth and flowering of gladiolus $\mathrm{cv}$. Jester. Annals of Plant Physiology, 16(2): 109-111.

Yadav, S. and Bhatia, S. K. (2018). Effect of plant growth regulators on sprouting, vegetative characters, flowering and corm production in Gladiolus sp. cv. Sancerre. International Journal of Pure Applied Bio Science, 6(2): 1142-1147.

\section{How to cite this article:}

Anant Ram Singh, Satendra Kumar, Rehan and Deepti Jha. 2021. Effect of Plant Growth Regulators (GA3, NAA and BA) on Growth and Flowering of Gladiolus (Gladiolus hybridus Hort.) cv. White Prosperity. Int.J.Curr.Microbiol.App.Sci. 10(02): 2008-2024.

doi: https://doi.org/10.20546/ijcmas.2021.1002.241 